\title{
The influence of neoadjuvant chemotherapy on complications of immediate DIEP flap breast reconstructions
}

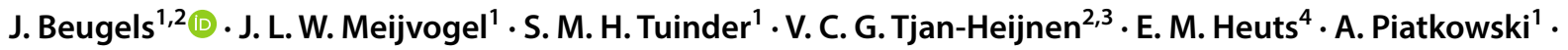 \\ R. R. W. J. van der Hulst ${ }^{1,2}$
}

Received: 7 September 2018 / Accepted: 15 April 2019 / Published online: 27 April 2019

(c) The Author(s) 2019

\begin{abstract}
Purpose The impact of neoadjuvant chemotherapy on the surgical outcomes of immediate breast reconstruction remains controversial. The aim of this study was to analyze the incidence of complications of immediate deep inferior epigastric artery perforator (DIEP) flap breast reconstructions in patients who received neoadjuvant chemotherapy compared to patients without neoadjuvant chemotherapy prior to surgery.

Methods A multicenter, retrospective cohort study was conducted of all patients who underwent immediate DIEP flap breast reconstruction between January 2010 and June 2017. Patients were divided in two groups as breast reconstructions with or without neoadjuvant chemotherapy, respectively. The primary outcome was the incidence of postoperative flap reexplorations, recipient-site complications and donor-site complications.

Results In total 432 immediate DIEP flap breast reconstructions in 326 patients were included. Forty-eight patients $(n=67$ flaps) received neoadjuvant chemotherapy prior to immediate breast reconstruction and 278 patients $(n=365$ flaps $)$ did not. No statistically significant differences for any major ( $4.5 \%$ vs. $10.4 \% ; p=0.175)$ or minor $(16.4 \%$ vs. $24.7 \% ; p=0.191)$ recipient-site complication were observed. Donor-site complications were recorded in $9(18.8 \%)$ and $62(22.2 \%)$ patients, respectively $(p=0.587)$. There was no difference in need for flap re-exploration between groups $(3.0 \%$ vs. $8.5 \% ; p=0.139)$. Correction for potential confounding variables did not result in significant differences.

Conclusions This study demonstrated similar complication rates for patients with and without neoadjuvant chemotherapy prior to immediate breast reconstruction, indicating that it is safe to perform an immediate DIEP flap breast reconstruction after neoadjuvant chemotherapy.
\end{abstract}

Keywords Neoadjuvant chemotherapy $\cdot$ DIEP flap $\cdot$ Breast reconstruction $\cdot$ Free flap $\cdot$ Complications $\cdot$ Microsurgery

Electronic supplementary material The online version of this article (https://doi.org/10.1007/s10549-019-05241-9) contains supplementary material, which is available to authorized users.

\section{J. Beugels}

jop.beugels@mumc.nl

1 Department of Plastic, Reconstructive and Hand Surgery, Maastricht University Medical Center, P.O. Box 5800, 6202 AZ Maastricht, The Netherlands

2 GROW - School for Oncology and Developmental Biology, Maastricht University, Maastricht, The Netherlands

3 Department of Medical Oncology, Maastricht University Medical Center, Maastricht, The Netherlands

4 Department of Surgery, Maastricht University Medical Center, Maastricht, The Netherlands

\section{Purpose}

Neoadjuvant chemotherapy is increasingly being used for the treatment of patients with breast cancer. It is defined as systemic therapy that is administered prior to locoregional treatment. Despite its initial role in the management of locally advanced breast cancer, the indications have been extended and it is now routinely used in early stage breast cancer treatment as well [1-3]. The survival and distant disease progression rates after neoadjuvant chemotherapy have been shown to be similar to adjuvant chemotherapy $[2$, 3]; however, neoadjuvant chemotherapy is associated with a moderately increased local recurrence risk [3]. Neoadjuvant chemotherapy allows early evaluation of the response to therapy and improves tumor resectability by downstaging the tumor [2-5]. This allows more breast-conserving 
surgery, with up to one-third of patients eligible for breastconserving surgery in whom mastectomy was initially indicated [3]. Hence, a mastectomy is still required in a considerable number of patients.

The postmastectomy reconstruction rates have increased over time and now trend toward more immediate bilateral implant-based breast reconstructions [6-8]. Nevertheless, autologous breast reconstructions provide a more natural and permanent result than implant-based reconstructions and offer higher patient satisfaction [9-11]. Of all options for autologous breast reconstruction, the deep inferior epigastric artery perforator (DIEP) flap is considered the first choice in most centers [12]. Immediate breast reconstruction, which yields better aesthetic results and may reduce psychological distress, can be offered to patients who are oncologically eligible [13, 14].

However, patients who receive neoadjuvant chemotherapy are less likely to undergo immediate reconstruction [15]. One of the reasons is that the impact of neoadjuvant chemotherapy on the surgical outcomes of immediate breast reconstructions remains controversial and highly variable results are reported in the literature [16-24]. In addition, all studies analyzed the total complication rates of either implant-based reconstructions [19], a combination of implant-based and autologous tissue reconstructions [21, 22], or a mix of several types of autologous reconstructions [16-18, 20, 24]. However, the complication rates are different for each type of breast reconstruction, regardless of the use of neoadjuvant chemotherapy; hence, they should be analyzed separately instead of being pooled. The aim of this study was to analyze the influence of neoadjuvant chemotherapy on the complication rates of immediate DIEP flap breast reconstructions only, by comparing the surgical outcomes of patients who received neoadjuvant chemotherapy to the outcomes of patients without neoadjuvant chemotherapy.

\section{Methods}

A retrospective cohort study was conducted based on a prospectively maintained database of all patients who underwent immediate DIEP flap breast reconstruction at Maastricht University Medical Center in the Netherlands and two community hospitals, VieCuri Medical Center Venlo and Zuyderland Medical Center Sittard-Geleen, between January 2010 and June 2017. All patients who received neoadjuvant chemotherapy prior to immediate DIEP flap breast reconstruction were identified. The control group consisted of all patients who underwent immediate DIEP flap breast reconstruction without neoadjuvant chemotherapy. The study was approved by the institutional review board and was performed in accordance with the ethical standards of the Declaration of Helsinki.
Patients over 18 years old were considered eligible if they underwent immediate DIEP flap breast reconstruction with or without neoadjuvant chemotherapy. Patients with bilateral breast reconstructions who had unilateral breast cancer and a contralateral prophylactic or riskreducing mastectomy were included, as well as patients who underwent bilateral risk-reducing mastectomy. However, patients with unilateral stacked DIEP flap breast reconstructions were excluded from this study due to the potential complications related to the complexity of the operation. All bilateral reconstructions were performed at the university hospital.

Neoadjuvant chemotherapy was offered to patients with stage III or early stage breast cancer when chemotherapy was already indicated by clinical stage and tumor characteristics. According to the hospital protocols, mastectomy and immediate breast reconstruction were planned approximately 5 weeks after the first day of the last chemotherapy cycle. Skin-sparing mastectomies were performed in all patients. Our surgical technique of DIEP flap breast reconstruction and the postoperative care has been published previously [25].

Patient characteristics (age, body mass index (BMI), comorbidities, smoking status), tumor characteristics, neoadjuvant chemotherapy regimens, time between the last chemotherapy cycle and surgery, adjuvant treatment, operative details (type of reconstruction, operative time, ischemia time), recipient- and donor-site complications, thromboembolic events, and flap re-explorations were recorded. Tumor characteristics included clinical TNM stage, histology, and hormone and HER2 receptor status. Complications were divided in major and minor complications. Major recipient-site complications were total flap loss, partial flap loss, and venous congestion. Minor recipientsite complications included infection, hematoma, seroma, fat necrosis, and wound problems. Minor donor-site complications were infection, hematoma, seroma, fat necrosis, wound problems, and bulging. Abdominal herniation confirmed by ultrasound was considered a major complication. Fat necrosis was defined as a palpable firmness identified by physical examination during postoperative evaluation or detected by ultrasound. Cases of partial flap loss were also registered as having fat necrosis. Pulmonary embolism and deep vein thrombosis (DVT) were considered as thromboembolic events. The follow-up duration was quantified as the time between the date of operation and the last visit to the outpatient clinic.

The primary outcome measure was the incidence of postoperative flap re-explorations, recipient-site complications, and donor-site complications following immediate DIEP flap breast reconstructions. Complication rates in patients who received neoadjuvant chemotherapy were compared to those of patients without neoadjuvant chemotherapy. 


\section{Data analysis}

Based on the distribution of data, continuous variables were presented as mean and standard deviation or as median and interquartile range (IQR). Categorical variables were reported as absolute numbers and percentages. The independent samples t test or the Mann-Whitney U test was used to compare continuous outcome variables as appropriate. Categorical data was tested with a Chi square or Fisher's exact test. The main unit of analysis was the flap. Because in a bilateral reconstruction both flaps are harvested from the same abdomen, standard comparative analyses cannot be performed as the assumption of independent data is violated. To account for clustered data in patients with a bilateral reconstruction, generalized estimating equations (GEE) with an exchangeable instead of independent structure were used to calculate the association between neoadjuvant chemotherapy and all recipient- and donor-site complications. Both unadjusted and adjusted odds ratios, corrected for clinically relevant variables (i.e., age, follow-up, and reason for mastectomy), were provided with its $95 \%$ confidence intervals. $P$ values $\leq 0.05$ were considered statistically significant. Statistical analyses were conducted using IBM SPSS (version 23.0, SPSS Inc., Chicago, Illinois, USA) for Windows.

\section{Results}

\section{Patient characteristics}

We included 326 eligible patients who underwent a total of 432 immediate DIEP flap breast reconstructions. Forty-eight patients ( $n=67$ flaps) received neoadjuvant chemotherapy prior to immediate breast reconstruction, whereas 278 patients ( $n=365$ flaps) underwent immediate DIEP flap breast reconstruction without neoadjuvant chemotherapy and served as a control group. The patient characteristics are presented in Table 1. Both groups were comparable with respect to BMI, comorbidities, type of reconstruction, and operative time for unilateral and bilateral reconstructions. Patients who received neoadjuvant chemotherapy were significantly younger $(46.3 \pm 8.1$ years vs. $51.3 \pm 9.3$ years; $p<0.001)$. Significantly more patients in the control group were active smokers (0\% vs. $9.4 \%$; $p=0.020)$ at the time of surgery. Median follow-up was 14 months (IQR 7 to 20 months) and 12 months (IQR 7 to 19 months) in the neoadjuvant chemotherapy and control group, respectively $(p=0.496)$.
Table 1 Patient characteristics ( $n=326$ patients)

\begin{tabular}{|c|c|c|c|}
\hline & $\begin{array}{l}\text { Neoadjuvant chemo- } \\
\text { therapy group } \\
n(\%)\end{array}$ & $\begin{array}{l}\text { Control group } \\
n(\%)\end{array}$ & $p$ value \\
\hline Total number of patients & 48 & 278 & \\
\hline Total number of immediate DIEP flaps & 67 & 365 & \\
\hline Age in years; mean $\pm \mathrm{SD}$ & $46.3 \pm 8.1$ & $51.3 \pm 9.3$ & $<0.001$ \\
\hline $\mathrm{BMI}$ in $\mathrm{kg} / \mathrm{m}^{2}$; mean $\pm \mathrm{SD}$ & $26.8 \pm 3.5$ & $26.7 \pm 3.8$ & 0.891 \\
\hline Active smoker & $0(0)$ & $26(9.4)$ & $0.020^{\mathrm{a}}$ \\
\hline Hypertension & $4(8.3)$ & $47(16.9)$ & 0.131 \\
\hline Diabetes mellitus & $1(2.1)$ & $14(5.0)$ & $0.707^{\mathrm{a}}$ \\
\hline \multicolumn{4}{|l|}{ Type of reconstruction } \\
\hline Unilateral & $29(60.4)$ & $191(68.7)$ & 0.258 \\
\hline Bilateral & $19(39.6)$ & $87(31.3)$ & \\
\hline \multicolumn{4}{|l|}{ Operative time in minutes; median (IQR) } \\
\hline Unilateral breast reconstructions & $394(349-448)$ & $387(331-450)$ & 0.929 \\
\hline Bilateral breast reconstructions & $468(409-534)$ & $484(413-574)$ & 0.574 \\
\hline Ischemia time in minutes; median $(\mathrm{IQR})^{\mathrm{b}}$ & $44(37-56)$ & $47(40-60)$ & 0.081 \\
\hline Flap weight in grams; mean $\pm \mathrm{SD}^{\mathrm{b}}$ & $612 \pm 248$ & $645 \pm 234$ & 0.304 \\
\hline Hospital stay in days; median (IQR) & $7(6-8)$ & $7(6-7)$ & 0.908 \\
\hline Follow-up in months; median (IQR) & $14(7-20)$ & $12(7-19)$ & 0.496 \\
\hline
\end{tabular}

$D I E P$ deep inferior epigastric artery perforator, $S D$ standard deviation, $B M I$ body mass index, $I Q R$ interquartile range

${ }^{a}$ Fisher's exact test was used

${ }^{\mathrm{b}}$ Total number of flaps as unit of analysis (neoadjuvant chemotherapy group: $n=67$; control group: $n=365$ ) 


\section{Oncological treatment and tumor characteristics}

An overview of the oncological treatment of the study population is presented in Table 2. Significantly more patients in the neoadjuvant chemotherapy group underwent mastectomy because of breast cancer compared to the control group in which roughly half of the patients underwent risk-reducing or prophylactic mastectomy $(p<0.001)$. Fifteen patients $(22.4 \%)$ in the neoadjuvant chemotherapy group underwent contralateral prophylactic mastectomy combined with an immediate bilateral DIEP flap breast reconstruction. Patients in the neoadjuvant chemotherapy group significantly more often received adjuvant endocrine therapy $(p=0.007)$ and immunotherapy $(p<0.001)$, whereas 45 patients in the control group received adjuvant chemotherapy compared to none in the neoadjuvant chemotherapy group $(p=0.001)$. The most frequently administered chemotherapy regimens were triplet chemotherapy consisting of docetaxel, adriamycin, and cyclophosphamide (TAC; $47.9 \%$ of patients), and a sequential chemotherapy of Adriamycin and cyclophosphamide followed by a taxane with or without HER2targeted therapy (35.4\% of patients). The time between the last chemotherapy cycle and surgery was a median of 4 weeks (IQR 3 to 6 weeks).
The tumor characteristics of all 432 breasts are shown in Table 3. Most patients who received neoadjuvant chemotherapy initially had stage II breast cancer, followed by highgrade stage I or stage III breast cancer. Also, more patients in the neoadjuvant chemotherapy group had triple negative tumors $(n=14 ; 26.9 \%)$. In a total of 21 out of 52 breasts (40.4\%) a pathological complete response to neoadjuvant chemotherapy was achieved and a near-complete response with a remainder of ductal carcinoma in situ (DCIS) in 3 breasts (5.8\%).

\section{Recipient-site complications}

Major and minor recipient-site complications were observed in 9.5\% (41/432 flaps) and 23.4\% (101/432 flaps) of flaps, with no significant differences between both groups (Table 4). No statistically significant differences in any of the major or minor postoperative complications were observed between patients who received neoadjuvant chemotherapy and patients with no neoadjuvant chemotherapy prior to immediate DIEP flap breast reconstruction. No total flap losses occurred in the neoadjuvant chemotherapy group versus 9 flap losses in the control group.

The odds ratios of the univariate analyses were adjusted for potential confounding variables (i.e., age in years,
Table 2 Oncological treatment $(n=326$ patients $)$

\begin{tabular}{llll}
\hline & $\begin{array}{l}\text { Neoadjuvant chemo- } \\
\text { therapy group } \\
n(\%)\end{array}$ & Control group & $p$ value \\
& & $n(\%)$ & \\
\hline Reason for mastectomy $^{\mathrm{a}}$ & $52(77.6)$ & $182(49.9)$ & $<0.001$ \\
Oncological & $15(22.4)$ & $183(50.1)$ & \\
Prophylactic & $10(20.8)$ & $66(23.7)$ & 0.660 \\
Genetic predisposition & $13(19.4)$ & $64(17.5)$ & 0.713 \\
History of lumpectomy & & \\
Adjuvant chemotherapy $_{\text {History of radiation therapy }}{ }^{\mathrm{a}}$ & $0(0)$ & $45(16.2)$ & $0.001^{\mathrm{b}}$ \\
Radiation therapy on DIEP flap $^{\mathrm{a}}$ & $9(13.4)$ & $55(15.1)$ & 0.729 \\
Endocrine therapy $^{\mathrm{a}}$ & $2(3.0)$ & $20(5.5)$ & $0.552^{\mathrm{b}}$ \\
Immunotherapy $_{\text {Neoadjuvant chemotherapy regimen }}$ & $26(54.2)$ & $94(33.8)$ & 0.007 \\
TAC & $18(37.5)$ & $25(9.0)$ & $<0.001$ \\
ACTH & & & - \\
AC-P & $23(47.9)$ & - & - \\
AC-T & $10(20.8)$ & - & - \\
Other & $5(10.4)$ & - & - \\
Time NAC-surgery in weeks; median (IQR) & $2(4.2)$ & - & - \\
\hline
\end{tabular}

$D I E P$ deep inferior epigastric artery perforator, $I Q R$ interquartile range, TAC Taxotere (Docetaxel), Adriamycin and Cyclophosphamide, ACTH Adriamycin, Cyclophosphamide, Taxol, and Herceptin, AC-P Adriamycin, Cyclophosphamide, and Taxol (Paclitaxel), AC-T Adriamycin, Cyclophosphamide, and Taxotere (Docetaxel), NAC neoadjuvant chemotherapy

${ }^{a}$ Total number of flaps as unit of analysis (neoadjuvant chemotherapy group: $n=67$; control group: $n=365$ )

${ }^{\mathrm{b}}$ Fisher's exact test was used 
Table 3 Tumor characteristics ( $n=432$ breasts)

\begin{tabular}{lcc}
\hline & $\begin{array}{l}\text { Neoadjuvant } \\
\text { chemotherapy } \\
\text { group }\end{array}$ & Control group \\
& $n(\%)$ & $n(\%)$ \\
\hline Breast pathology & & \\
No cancer & $15(22.4)$ & $181(49.6)$ \\
DCIS/LCIS & $0(0)$ & $53(14.5)$ \\
Invasive carcinoma & $52(77.6)$ & $129(35.3)$ \\
Other cancer & $0(0)$ & $2(0.5)$ \\
Breast cancer stage/clinical TNM stage & \\
Stage I & $10(19.2)$ & $63(48.8)$ \\
Stage IIa & $31(59.7)$ & $30(23.3)$ \\
Stage IIb & $9(17.3)$ & $23(17.8)$ \\
Stage IIIa & $1(1.9)$ & $9(7.0)$ \\
Stage IIIb & $1(1.9)$ & $0(0)$ \\
Stage IIIc & $0(0)$ & $1(0.8)$ \\
Stage IV & $0(0)$ & $3(2.3)$ \\
Receptor status & & $102(79.1)$ \\
Estrogen receptor positive & $34(65.4)$ & $85(65.9)$ \\
Progesterone receptor positive & $25(48.1)$ & $21(16.3)$ \\
Her2/Neu positive & $18(34.6)$ & $15(11.6)$ \\
Triple negative & $14(26.9)$ & \\
\hline
\end{tabular}

DCIS ductal carcinoma in situ, LCIS lobular carcinoma in situ

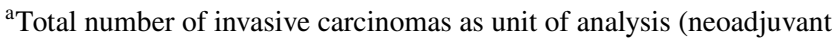
chemotherapy group: $n=52$; control group: $n=129$ )

follow-up in months, and either oncological or prophylactic reason for mastectomy) in multivariable models. Again, no statistically significant differences in adjusted odds ratios were registered for any of the major or minor complications. Univariate and multivariable analyses of recipient-site complications with the patient instead of the flap as the unit of analysis showed similar results (see additional data "Online Resource 1: Table 1").

Lastly, in one patient $(2.1 \%)$ who received neoadjuvant chemotherapy a pulmonary embolism was diagnosed postoperatively, whereas a thromboembolic event was documented in four patients $(1.4 \% ; 3$ pulmonary embolisms and one deep venous thrombosis) in the control group (unadjusted OR 1.46, 95\% CI 0.16-13.3, $p=0.739$; adjusted OR $2.59,95 \%$ CI $0.20-34.3, p=0.471)$.

\section{Donor-site complications}

Abdominal herniation as a major donor-site complication was only recorded once $(0.4 \%)$ in the control group (Table 5). Minor donor-site complications were observed in $9(18.8 \%)$ and $61(21.9 \%)$ patients, respectively, with no significant differences between groups $(p=0.619)$. Of all minor complications, wound healing problems were most frequently documented in both groups $(16.7 \%$ vs. $16.2 \%$, OR $1.04,95 \%$ CI $0.46-2.36, p=0.934)$. After adjusting the odds ratios of the univariate analyses for the aforementioned potential confounding variables comparable results were found.

\section{Flap re-explorations}

An overview of the flap re-explorations is presented in Table 6. In total, 33 DIEP flaps $(7.6 \% ; 2 / 67$ in the neoadjuvant chemotherapy group vs. $31 / 365$ in the control group) required re-exploration and 21 flaps $(4.9 \% ; 2 / 67$ in the neoadjuvant chemotherapy group vs. $19 / 365$ in the control group) required reanastomosis of the vein and/or

Table 4 Recipient-site complications ( $n=432$ flaps)

\begin{tabular}{lccllll}
\hline & $\begin{array}{l}\text { NAC } \\
(n=67)\end{array}$ & $\begin{array}{l}\text { Control } \\
(n=365)\end{array}$ & OR $(95 \%$ CI $)$ & $p$ value & ${\text { Adjusted OR }(95 \% \text { CI })^{\mathrm{a}}}^{\text {Adjusted } p \text { value }^{\mathrm{a}}}$ \\
& $n(\%)$ & $n(\%)$ & & & & \\
\hline Major complication $(\geq 1)$ & $3(4.5)$ & $38(10.4)$ & $0.43(0.13-1.46)$ & 0.175 & $0.39(0.11-1.32)$ & 0.129 \\
Total flap loss & $0(0)$ & $9(2.5)$ & - & $0.366^{\mathrm{b}}$ & - & - \\
Partial flap loss & $2(3.0)$ & $15(4.1)$ & $0.78(0.17-3.58)$ & 0.754 & $0.73(0.15-3.70)$ & 0.706 \\
Venous congestion & $1(1.5)$ & $20(5.5)$ & $0.28(0.04-2.16)$ & 0.223 & $0.21(0.03-1.61)$ & 0.133 \\
Minor complication $(\geq 1)$ & $11(16.4)$ & $90(24.7)$ & $0.61(0.30-1.28)$ & 0.191 & $0.59(0.28-1.22)$ & 0.151 \\
Infection & $3(4.5)$ & $24(6.6)$ & $0.70(0.20-2.41)$ & 0.566 & $0.69(0.20-2.42)$ & 0.560 \\
Hematoma & $3(4.5)$ & $34(9.3)$ & $0.47(0.14-1.58)$ & 0.221 & $0.40(0.12-1.39)$ & 0.149 \\
Seroma & $0(0)$ & $12(3.3)$ & - & $0.227^{\mathrm{b}}$ & - & - \\
Fat necrosis & $4(6.0)$ & $39(10.7)$ & $0.53(0.19-1.52)$ & 0.237 & $0.51(0.18-1.49)$ & 0.220 \\
Wound problems & $5(7.5)$ & $35(9.6)$ & $0.81(0.27-2.45)$ & 0.714 & $0.81(0.27-2.46)$ & 0.709 \\
\hline
\end{tabular}

$N A C$ neoadjuvant chemotherapy, $O R$ odds ratio, $C I$ confidence interval

${ }^{a}$ Adjusted for age (years), follow-up (months), and reason for mastectomy (oncological vs. prophylactic)

${ }^{\text {b}}$ Fisher's exact test was used 
Table 5 Donor-site complications $(n=326$ patients)

\begin{tabular}{|c|c|c|c|c|c|c|}
\hline & $\begin{array}{l}\text { NAC } \\
(n=48) \\
n(\%)\end{array}$ & $\begin{array}{l}\text { Control } \\
(n=278) \\
n(\%)\end{array}$ & OR $(95 \% \mathrm{CI})$ & $p$ value & Adjusted OR $(95 \% \mathrm{CI})^{\mathrm{a}}$ & Adjusted $p$ value ${ }^{\mathrm{a}}$ \\
\hline Major complication $(\geq 1)$ & $0(0)$ & $1(0.4)$ & - & $1.000^{\mathrm{b}}$ & - & - \\
\hline Herniation & $0(0)$ & $1(0.4)$ & - & $1.000^{\mathrm{b}}$ & - & - \\
\hline Minor complication $(\geq 1)$ & $9(18.8)$ & $61(21.9)$ & $0.82(0.38-1.79)$ & 0.619 & $0.92(0.40-2.12)$ & 0.841 \\
\hline Infection & $4(8.3)$ & $17(6.1)$ & $1.40(0.45-4.34)$ & 0.565 & $1.84(0.55-6.18)$ & 0.323 \\
\hline Hematoma & $0(0)$ & $3(1.1)$ & - & $1.000^{\mathrm{b}}$ & - & - \\
\hline Seroma & $0(0)$ & $10(3.6)$ & - & $0.368^{\mathrm{b}}$ & - & - \\
\hline Fat necrosis & $0(0)$ & $16(5.8)$ & - & $0.142^{\mathrm{b}}$ & - & - \\
\hline Bulging & $0(0)$ & $2(0.7)$ & - & $1.000^{\mathrm{b}}$ & - & - \\
\hline Wound problems & $8(16.7)$ & $45(16.2)$ & $1.04(0.46-2.36)$ & 0.934 & $1.25(0.51-3.04)$ & 0.628 \\
\hline
\end{tabular}

$N A C$ neoadjuvant chemotherapy, $O R$ odds ratio, $C I$ confidence interval

${ }^{a}$ Adjusted for age (years), follow-up (months), and reason for mastectomy (oncological vs. prophylactic)

${ }^{\mathrm{b}}$ Fisher's exact test was used

\section{Discussion}

Table 6 Flap re-explorations ( $n=432$ flaps)

\begin{tabular}{llll}
\hline & $\begin{array}{l}\text { NAC } \\
(n=67)\end{array}$ & $\begin{array}{l}\text { Control } \\
(n=365)\end{array}$ & $p$ value \\
& $n(\%)$ & $n(\%)$ & \\
\hline Re-exploration & $2(3.0)$ & $31(8.5)$ & $0.139^{\mathrm{b}}$ \\
Reanastomosis & $2(3.0)$ & $19(5.2)$ & $0.756^{\mathrm{b}}$ \\
Reason re-exploration & & & \\
Arterial insufficiency & $1(1.5)$ & $6(1.6)$ & $1.000^{\mathrm{b}}$ \\
Venous insufficiency & $2(3.0)$ & $21(5.8)$ & $0.554^{\mathrm{b}}$ \\
Hematoma & $0(0)$ & $7(1.9)$ & $0.602^{\mathrm{b}}$ \\
Kinking & $0(0)$ & $6(1.6)$ & $0.596^{\mathrm{b}}$ \\
Result re-exploration & & & \\
Viable flap & $2(100.0)$ & $19(61.3)$ & $0.523^{\mathrm{b}}$ \\
Partial flap loss & $0(0)$ & $3(9.7)$ & $1.000^{\mathrm{b}}$ \\
Total flap loss & $0(0)$ & $9(29.0)$ & $1.000^{\mathrm{b}}$ \\
\hline
\end{tabular}

$N A C$ neoadjuvant chemotherapy

${ }^{\mathrm{a}}$ As a percentage of the total flaps that required re-exploration (NAC group: $n=2$; control group: $n=31$ )

${ }^{\mathrm{b}}$ Fisher's exact test was used

artery, with no significant differences between groups. The most frequent reason for re-exploration of the flap was venous congestion, which was comparable for both groups. Re-exploration of the flap resulted in viable flaps in both cases in the neoadjuvant chemotherapy group and in $61.3 \%$ (19/31 flaps) in the control group. Analysis of flap re-explorations with the patient as the unit of analysis also showed similar results (see additional data "Online Resource 1: Table 2").
The aim of this study was to evaluate the influence of neoadjuvant chemotherapy on the complications of immediate DIEP flap breast reconstructions.

Direct comparison of the surgical outcomes of patients with and without neoadjuvant chemotherapy prior to immediate breast reconstruction demonstrated similar complication rates. No statistically significant differences were found between both groups for any major or minor recipient- or donor-site complication, nor for the re-exploration rate of DIEP flap breast reconstructions, also after adjusting for potential confounding variables. Additionally, no delay in adjuvant treatment was documented in the neoadjuvant chemotherapy group.

Our findings are in line with a number of previous studies that showed a similar complication rate irrespective of neoadjuvant chemotherapy use [18-22, 24]. A prospective study by Schaverien et al. [18]. included 22 patients with neoadjuvant chemotherapy and 41 patients without neoadjuvant chemotherapy, who had undergone immediate DIEP flap breast reconstruction. There were no significant differences in postoperative complication rates (67\% vs. $65 \% ; p=0.87)$ or need for reoperations $(17 \%$ vs. $9 \% ; p=0.30$ ), although three patients in each group had a delay in adjuvant treatment. An interesting point raised by the authors is that the dissection of the perforators seemed to be more difficult in patients who received neoadjuvant chemotherapy. This experience is shared by us, as we feel that chemotherapy might affect the tissue quality and structure, even though we did not find a significant difference in operative time and ischemia time. Zweiffel-Schlatter et al. [20]. also found no significant differences in complication rates ( $36 \%$ vs. $29 \%$ ) in 47 patients 
with neoadjuvant chemotherapy compared to 52 patients without neoadjuvant chemotherapy who underwent various types of autologous breast reconstructions. A retrospective study by Azzawi et al. [21]. in a mixed group of patients who underwent either autologous or implantbased breast reconstruction also showed that neoadjuvant chemotherapy did not increase the risk of postoperative complications even though three-quarters of the patients treated with neoadjuvant chemotherapy and a quarter of the controls received adjuvant radiation therapy.

Conversely, some studies reported that neoadjuvant chemotherapy was associated with an increased risk of complications [16, 17]. In a large study by Mehrara et al. [16], 952 patients were included who underwent immediate autologous breast reconstruction, of which 70 patients (7.4\%) received neoadjuvant chemotherapy. Neoadjuvant chemotherapy came out as an independent predictor of postoperative complications (OR $2.1 ; p<0.01$ ) and was associated with (donor-site) wound healing problems (OR 2.9; $p=0.02$ ) and fat necrosis (OR 2.8; $p<0.01$ ) in multivariate analysis. However, this study did not include DIEP flaps, but mainly transverse rectus abdominis (TRAM) flap breast reconstructions besides other flaps with different complication rates for each type of breast reconstruction. Obesity was a significant predictor of overall complications which is in line with findings of our previous studies $[25,26]$. A study by Albino et al. [17]. included patients who underwent immediate autologous breast reconstruction followed by radiation therapy and reported that neoadjuvant chemotherapy increased the incidence of complications (OR 4.4; $p=0.04$ ), and more specifically skin complications (OR 2.4; $p=0.01$ ).

Surprisingly, a meta-analysis by Song et al. [23]. demonstrated that patients treated with neoadjuvant chemotherapy were even less likely to have complications after immediate breast reconstruction. This conclusion, however, was based on the analysis of a combination of various types of breast reconstruction techniques, ranging from autologous to implant-based reconstruction. No subgroup analysis was done for the specific breast reconstruction techniques, and the results were not adjusted for confounding variables, which makes the results difficult to interpret. Additionally, the meta-analysis did not include the study by Mehrara et al. [16]. that reported an increased risk of complications after neoadjuvant chemotherapy. Also in our study less postoperative complications were observed in patients who received neoadjuvant chemotherapy, even though this trend was not statistically significant, possibly due to the relatively small sample size. Mechanisms behind the protective association of neoadjuvant chemotherapy still remain unknown [23, 27]. In agreement with other authors [19,23], the selection of patients might have contributed to this observation, as for example patients who received neoadjuvant chemotherapy were significantly younger and more patients in the control group were active smokers.

Limitations of this study include the retrospective study design and the potential bias that are associated with it. Two patient cohorts were constructed which inevitably led to selection bias since patients who had an indication for neoadjuvant chemotherapy initially had a less favorable clinical tumor stage whereas also patients without breast cancer who underwent risk-reducing surgery were included in the control group. Even though this is the largest study to date on specifically DIEP flap breast reconstructions, the sample size is still relatively small and may have influenced the results. No subgroup analysis was performed to assess the effects of time in weeks between the last dose of chemotherapy and surgery. Strengths of this study, however, are the comprehensive analysis of all complications and the multicenter design, which resulted in different oncological treatment plans.

\section{Conclusions}

This study demonstrated similar complication rates for patients with and without neoadjuvant chemotherapy prior to immediate breast reconstruction, indicating that it is safe to perform an immediate DIEP flap breast reconstruction after neoadjuvant chemotherapy. There were no statistically significant differences in the incidences of flap re-explorations, recipient-site complications or donor-site complications. More prospective data is required to confirm these results.

\section{Compliance with ethical standards}

Conflict of interest All authors declare that they have no conflicts of interest.

Ethical approval All procedures performed in studies involving human participants were in accordance with the ethical standards of the institutional research committee and with the 1964 Helsinki Declaration and its later amendments or comparable ethical standards. This article does not contain any studies with animals performed by any of the authors.

Informed consent Informed consent was obtained from all individual participants included in the study.

Open Access This article is distributed under the terms of the Creative Commons Attribution 4.0 International License (http://creativeco mmons.org/licenses/by/4.0/), which permits unrestricted use, distribution, and reproduction in any medium, provided you give appropriate credit to the original author(s) and the source, provide a link to the Creative Commons license, and indicate if changes were made. 


\section{References}

1. Smith IE, Lipton L (2001) Preoperative/neoadjuvant medical therapy for early breast cancer. Lancet Oncol 2(9):561-570. https ://doi.org/10.1016/S1470-2045(01)00490-9

2. Mieog JS, van der Hage JA, van de Velde CJ (2007) Preoperative chemotherapy for women with operable breast cancer. Cochrane Database Syst Rev. https://doi.org/10.1002/14651858.cd005002. pub2

3. Early Breast Cancer Trialists' Collaborative G (2018) Longterm outcomes for neoadjuvant versus adjuvant chemotherapy in early breast cancer: meta-analysis of individual patient data from ten randomised trials. Lancet Oncol 19(1):27-39. https:// doi.org/10.1016/S1470-2045(17)30777-5

4. Liu SV, Melstrom L, Yao K, Russell CA, Sener SF (2010) Neoadjuvant therapy for breast cancer. J Surg Oncol 101(4):283-291. https://doi.org/10.1002/jso.21446

5. Van de Wiel M, Dockx Y, Van den Wyngaert T, Stroobants S, Tjalma WA, Huizing MT (2016) Neoadjuvant systemic therapy in breast cancer: challenges and uncertainties. Eur J Obstet Gynecol Reprod Biol 210:144-156. https://doi.org/10.1016/j. ejogrb.2016.12.014

6. Cemal Y, Albornoz CR, Disa JJ, McCarthy CM, Mehrara BJ, Pusic AL, Cordeiro PG, Matros E (2013) A paradigm shift in US breast reconstruction: Part 2. The influence of changing mastectomy patterns on reconstructive rate and method. Plast Reconstr Surg 131(3):320e-326e. https://doi.org/10.1097/prs.0b013e3182 7 cf576

7. Lang JE, Summers DE, Cui H, Carey JN, Viscusi RK, Hurst CA, Waer AL, Ley ML, Sener SF, Vijayasekaran A (2013) Trends in post-mastectomy reconstruction: a SEER database analysis. J Surg Oncol 108(3):163-168. https://doi.org/10.1002/jso.23365

8. Jagsi R, Jiang J, Momoh AO, Alderman A, Giordano SH, Buchholz TA, Kronowitz SJ, Smith BD (2014) Trends and variation in use of breast reconstruction in patients with breast cancer undergoing mastectomy in the United States. J Clin Oncol 32(9):919926. https://doi.org/10.1200/JCO.2013.52.2284

9. Hu ES, Pusic AL, Waljee JF, Kuhn L, Hawley ST, Wilkins E, Alderman AK (2009) Patient-reported aesthetic satisfaction with breast reconstruction during the long-term survivorship Period. Plast Reconstr Surg 124(1):1-8. https://doi.org/10.1097/ PRS.0b013e3181ab10b2

10. Yueh JH, Slavin SA, Adesiyun T, Nyame TT, Gautam S, Morris DJ, Tobias AM, Lee BT (2010) Patient satisfaction in postmastectomy breast reconstruction: a comparative evaluation of DIEP, TRAM, latissimus flap, and implant techniques. Plast Reconstr Surg 125(6):1585-1595. https://doi.org/10.1097/PRS.0b013e3181 cb6351

11. Craft RO, Colakoglu S, Curtis MS, Yueh JH, Lee BS, Tobias AM, Lee BT (2011) Patient satisfaction in unilateral and bilateral breast reconstruction [outcomes article]. Plast Reconstr Surg 127(4):1417-1424. https://doi.org/10.1097/PRS.0b013e3182 $08 \mathrm{~d} 12 \mathrm{a}$

12. Healy C, Allen RJ Sr (2014) The evolution of perforator flap breast reconstruction: twenty years after the first DIEP flap. J Reconstr Microsurg 30(2):121-125. https://doi.org/10.1055/s-0033-13572 72

13. Zhong T, Hu J, Bagher S, Vo A, O’Neill AC, Butler K, Novak CB, Hofer SO, Metcalfe KA (2016) A comparison of psychological response, body image, sexuality, and quality of life between immediate and delayed autologous tissue breast reconstruction: a prospective long-term outcome study. Plast Reconstr Surg 138(4):772-780. https://doi.org/10.1097/PRS.0000000000002536

14. Al-Ghazal SK, Sully L, Fallowfield L, Blamey RW (2000) The psychological impact of immediate rather than delayed breast reconstruction. Eur J Surg Oncol 26(1):17-19

15. Hu YY, Weeks CM, In H, Dodgion CM, Golshan M, Chun YS, Hassett MJ, Corso KA, Gu X, Lipsitz SR, Greenberg CC (2011) Impact of neoadjuvant chemotherapy on breast reconstruction. Cancer 117(13):2833-2841. https://doi.org/10.1002/cncr.25872

16. Mehrara BJ, Santoro TD, Arcilla E, Watson JP, Shaw WW, Da Lio AL (2006) Complications after microvascular breast reconstruction: experience with 1195 flaps. Plast Reconstr Surg 118(5):1100-1109. https://doi.org/10.1097/01.prs.0000236898 .87398.d6 (discussion 1110-1101)

17. Albino FP, Koltz PF, Ling MN, Langstein HN (2010) Irradiated autologous breast reconstructions: effects of patient factors and treatment variables. Plast Reconstr Surg 126(1):12-16. https://doi. org/10.1097/PRS.0b013e3181da878f

18. Schaverien MV, Munnoch DA (2013) Effect of neoadjuvant chemotherapy on outcomes of immediate free autologous breast reconstruction. Eur J Surg Oncol 39(5):430-436. https://doi. org/10.1016/j.ejso.2013.02.015

19. Donker M, Hage JJ, Woerdeman LA, Rutgers EJ, Sonke GS, Vrancken Peeters MJ (2012) Surgical complications of skin sparing mastectomy and immediate prosthetic reconstruction after neoadjuvant chemotherapy for invasive breast cancer. Eur J Surg Oncol 38(1):25-30. https://doi.org/10.1016/j.ejso.2011.09.005

20. Zweifel-Schlatter M, Darhouse N, Roblin P, Ross D, Zweifel M, Farhadi J (2010) Immediate microvascular breast reconstruction after neoadjuvant chemotherapy: complication rates and effect on start of adjuvant treatment. Ann Surg Oncol 17(11):2945-2950. https://doi.org/10.1245/s10434-010-1195-9

21. Azzawi K, Ismail A, Earl H, Forouhi P, Malata CM (2010) Influence of neoadjuvant chemotherapy on outcomes of immediate breast reconstruction. Plast Reconstr Surg 126(1):1-11. https:// doi.org/10.1097/PRS.0b013e3181da8699

22. Warren Peled A, Itakura K, Foster RD, Hamolsky D, Tanaka J, Ewing C, Alvarado M, Esserman LJ, Hwang ES (2010) Impact of chemotherapy on postoperative complications after mastectomy and immediate breast reconstruction. Arch Surg 145(9):880-885. https://doi.org/10.1001/archsurg.2010.163

23. Song J, Zhang X, Liu Q, Peng J, Liang X, Shen Y, Liu H, Li H (2014) Impact of neoadjuvant chemotherapy on immediate breast reconstruction: a meta-analysis. PLoS ONE 9(5):e98225. https:// doi.org/10.1371/journal.pone.0098225

24. Narui K, Ishikawa T, Satake T, Adachi S, Yamada A, Shimada K, Shimizu D, Kida K, Sugae S, Ichikawa Y, Tanabe M, Sasaki T, Endo I (2015) Outcomes of immediate perforator flap reconstruction after skin-sparing mastectomy following neoadjuvant chemotherapy. Eur J Surg Oncol 41(1):94-99. https://doi.org/10.1016/j. ejso.2014.09.001

25. Beugels J, Hoekstra LT, Tuinder SM, Heuts EM, van der Hulst RR, Piatkowski AA (2016) Complications in unilateral versus bilateral deep inferior epigastric artery perforator flap breast reconstructions: a multicentre study. J Plast Reconstr Aesthet Surg 69(9):1291-1298. https://doi.org/10.1016/j.bjps.2016.04.010

26. Beugels J, Bod L, van Kuijk SMJ, Qiu SS, Tuinder SMH, Heuts EM, Piatkowski A, van der Hulst R (2018) Complications following immediate compared to delayed deep inferior epigastric artery perforator flap breast reconstructions. Breast Cancer Res Treat 169(2):349-357. https://doi.org/10.1007/s10549-018-4695-0 
27. Abt NB, Flores JM, Baltodano PA, Sarhane KA, Abreu FM, Cooney CM, Manahan MA, Stearns V, Makary MA, Rosson GD (2014) Neoadjuvant chemotherapy and short-term morbidity in patients undergoing mastectomy with and without breast reconstruction. JAMA Surg 149(10):1068-1076. https://doi. org/10.1001/jamasurg.2014.1076
Publisher's Note Springer Nature remains neutral with regard to jurisdictional claims in published maps and institutional affiliations. 